\title{
REDUCTIONS \\ OF LOCALLY CONFORMAL SYMPLECTIC STRUCTURES AND DE RHAM COHOMOLOGY TANGENT TO A FOLIATION
}

\author{
WOJCIECH DOMITRZ \\ Faculty of Mathematics and Information Science, Warsaw University of Technology \\ Plac Politechniki 1, 00-661 Warszawa, Poland \\ E-mail: domitrz@mini.pw.edu.pl
}

\begin{abstract}
We define a procedure of reduction of locally conformal symplectic structures. We find a necessary and sufficient condition for this reduction to hold in terms of a special kind of de Rham cohomology class (tangent to the characteristic foliation) of the Lee form.
\end{abstract}

1. Introduction. Let $M$ be a smooth even-dimensional manifold, $\operatorname{dim} M=2 n>2$. Let $\Omega$ be a smooth nondegenerate 2 -form on $M$. If there exist an open cover $\left\{U_{a}: a \in A\right\}$ of $M$ and smooth positive functions $f_{a}$ on $U_{a}$ such that

$$
\Omega_{a}=\left.f_{a} \Omega\right|_{U_{a}}
$$

is a symplectic form on $U_{a}$ for $a \in A$ then $\Omega$ is called a locally conformal symplectic form.

Equivalently (see [11]) $\Omega$ satisfies the following condition:

$$
d \Omega=\omega \wedge \Omega,
$$

where $\omega$ is a closed 1-form. $\omega$ is uniquely determined by $\Omega$ and is called the Lee form of $\Omega$. $(M, \Omega, \omega)$ is called a locally conformal symplectic manifold.

If $\Omega$ satisfies (1) then $\left.\omega\right|_{U_{a}}=d\left(\ln f_{a}\right)$ for all $a \in A$. If $f_{a}$ is constant for all $a \in A$ then $\Omega$ is a symplectic form on $M$. The Lee form of the symplectic form is obviously zero.

Locally conformal symplectic manifolds are generalized phase spaces of Hamiltonian dynamical systems since the form of the Hamiltonian equations is preserved by homothetic canonical transformations [17].

2000 Mathematics Subject Classification: Primary 53D20; Secondary 53C12, 14F40.

Key words and phrases: locally conformal symplectic manifolds, symplectic reduction, de Rham cohomology.

Research supported by Institute of Mathematics, Polish Academy of Sciences.

The paper is in final form and no version of it will be published elsewhere. 
Two locally conformal symplectic forms $\Omega_{1}$ and $\Omega_{2}$ on $M$ are conformally equivalent if $\Omega_{2}=f \Omega_{1}$ for some smooth positive function $f$ on $M$. A conformal equivalence class of locally conformal symplectic forms on $M$ is a locally conformal symplectic structure on $M([3])$.

Let $Q$ be a smooth submanifold of $M$. Let $\iota: Q \hookrightarrow M$ denote the standard inclusion. We say that two locally conformal symplectic forms $\Omega_{1}$ and $\Omega_{2}$ on $M$ are conformally equivalent on $Q$ if $\iota^{*} \Omega_{2}=f \iota^{*} \Omega_{1}$ for some smooth positive function $f$ on $Q$.

Clearly the Lee form of a locally conformal symplectic form is exact if and only if $\Omega$ is conformally equivalent to a symplectic form [17]. Then the locally conformal symplectic structure is globally conformal symplectic.

Locally conformal symplectic forms were introduced by Lee [11]. They have been intensively studied in [17], [8], [9], [10], [3].

In [18] the symmetry of the Lyapunov spectrum in locally conformal Hamiltonian systems was studied. It was shown that Gaussian isokinetic dynamics, Nosé-Hoovers dynamics and other systems can be treated as locally conformal Hamiltonian systems. A kind of reduction was applied to obtain these results.

In [10] (see Section 3) a reduction procedure of a locally conformal symplectic form is defined using the general definition of reduction (see [12]). But the conditions for reduction of locally conformal symplectic form are very restrictive (see Proposition 1 in [10] and Proposition 4 in Section 4). There are local obstructions, a locally conformal symplectic form on a germ of a generic smooth hypersurface cannot be reduced using this procedure (see Example 1). The procedure of reduction of a locally conformal symplectic form has no application to reduction of systems with symmetry defined in Section 5 of $[10]$.

In this paper we show a different approach to this problem. We propose to reduce a locally conformal symplectic structure (the conformal equivalence class of a locally conformal symplectic form) instead of a locally conformal symplectic form. This procedure of reduction can be applied to a much wider class of submanifolds. There are no local obstructions to this procedure. But there are global obstructions. We find a necessary and sufficient condition for this reduction to hold in terms of a special kind of de Rham cohomology class (tangent to the characteristic foliation) of the Lee form.

2. De Rham cohomology tangent to a foliation. Let $Q$ be a smooth manifold and let $\mathcal{F}$ be a foliation in $Q$. We denote by $\Omega^{p}(Q)$ the space of differential $p$-forms on $Q$. By $\Omega^{p}(Q, \mathcal{F})$ we denote the space of $p$-forms $\omega$ satisfying the following condition:

$$
\left.\omega\right|_{q}\left(v_{1}, \ldots, v_{p}\right)=0
$$

for any $q \in Q$ and for any vectors $v_{1}, \ldots, v_{p}$ tangent to the foliation $\mathcal{F}$ at $q$. It means that $\omega \in \Omega^{p}(Q, \mathcal{F})$ if and only if $\iota_{q}^{*} \omega=0$ for any $q \in Q$, where $i_{q}: \mathcal{F}_{q} \hookrightarrow Q$ is the standard inclusion of the leaf $\mathcal{F}_{q}$ of the foliation $\mathcal{F}$ into $Q$.

$\Omega^{p}(Q, \mathcal{F})$ is a subcomplex of the de Rham complex $\Omega^{*}(Q)$. This follows from the relation $\iota_{q}^{*} d \omega=d \iota_{q}^{*} \omega$. 
We define the factor space

$$
\Omega^{p}(\mathcal{F})=\Omega^{p}(Q) / \Omega^{p}(Q, \mathcal{F}) .
$$

The operator $d_{p}: \Omega^{p}(\mathcal{F}) \rightarrow \Omega^{p+1}(\mathcal{F}), d_{p}(\omega)=d \omega$ is well defined since $d \Omega^{p}(Q, \mathcal{F}) \subset$ $\Omega^{p+1}(Q, \mathcal{F})$. Therefore one has the following differential complex

$$
\left(\Omega^{*}(\mathcal{F}), d\right): \Omega^{0}(\mathcal{F}) \rightarrow^{d_{0}} \Omega^{1}(\mathcal{F}) \rightarrow^{d_{1}} \Omega^{2}(\mathcal{F}) \rightarrow^{d_{2}} \ldots
$$

The cohomology of this complex

$$
H^{p}(\mathcal{F})=H^{p}\left(\Omega^{*}(\mathcal{F}), d\right)=\operatorname{ker} d_{p} / \operatorname{im} d_{p-1}
$$

is called the de Rham cohomology tangent to foliation $\mathcal{F}$ (see [16] for a similar construction).

Directly from the definition of the cohomology $H^{p}(\mathcal{F})$ we get the following propositions.

Proposition 1. $H^{p}(\mathcal{F})=0$ for $p$ greater than the dimension of the leaves of the foliation $\mathcal{F}$.

Proposition 2. Let $\omega \in \Omega^{p}(Q)$ such that $d \omega \in \Omega^{p+1}(Q, \mathcal{F})$. If $[\omega]=0$ in $H^{p}(\mathcal{F})$ then $\left[\iota_{q}^{*} \omega\right]=0$ in $H^{p}\left(\mathcal{F}_{q}\right)$ for any $q \in Q$.

We define a special kind of contractions along a foliation.

DEFINITION 1. We say that $Q$ is contractible to a submanifold $S$ along the foliation $\mathcal{F}$ if there exists a family of maps $F_{t}: Q \rightarrow Q, t \in[0,1]$ which is (piecewise) smooth in $t$, such that $F_{1}$ is the identity map, $F_{0}(Q) \subset S,\left.F_{0}\right|_{S}=i d_{S}$ and $\forall q \in Q F_{t}\left(\mathcal{F}_{q}\right) \subset \mathcal{F}_{q}$ for all $t \in[0,1]$. We call the family $F_{t}$ a (piecewise) smooth contraction of $Q$ to $S$ along the foliation $\mathcal{F}$.

Using the analog of the homotopy operator for the above contraction we prove the following theorem.

THEOREM 1. Let $S$ be a smooth submanifold of $Q$ transversal to a foliation $\mathcal{F}$. If $Q$ is contractible to $S$ along the foliation $\mathcal{F}$ then the cohomology groups $H^{p}(\mathcal{F})$ and $H^{p}(\mathcal{F} \cap S)$ are isomorphic.

Proof. Since $\mathcal{F}$ is transversal to $S$ then $\mathcal{F} \cap S$ is a foliation on $S$. Let $F_{t}$ be a (piecewise) smooth contraction of $Q$ to $S$ along the foliation $\mathcal{F}$. Let $\omega$ be a $p$-form on $Q$ such that $d \omega \in \Omega^{p+1}(Q, \mathcal{F})$ and $i: S \hookrightarrow Q$ be the standard inclusion of $S$ in $Q$. Then

$$
\begin{aligned}
\omega-\left(F_{0}^{*} \circ \iota^{*}\right)(\omega) & =F_{1}^{*} \omega-F_{0}^{*} \omega=\int_{0}^{1} \frac{d}{d t} F_{t}^{*} \omega d t=\int_{0}^{1} F_{t}^{*}\left(\mathcal{L}_{V_{t}} \omega\right) d t \\
& \left.\left.\left.\left.=\int_{0}^{1} F_{t}^{*}\left(V_{t}\right\rfloor d \omega+d\left(V_{t}\right\rfloor \omega\right)\right) d t=\int_{0}^{1}\left[F_{t}^{*}\left(V_{t}\right\rfloor d \omega\right)+d\left(F_{t}^{*}\left(V_{t}\right\rfloor \omega\right)\right)\right] d t
\end{aligned}
$$

where $V_{t} \circ F_{t}=\frac{d F_{t}}{d t}$ and $\mathcal{L}_{V_{t}}$ is the Lie derivative along the vector field $V_{t}$. 
For any $q \in Q$ and any $\left(u_{1}, \ldots, u_{p}\right)$ tangent to $\mathcal{F}_{q}$ we have

$$
\begin{aligned}
\left.\left.\int_{0}^{1} F_{t}^{*}\left(V_{t}\right\rfloor d \omega\right) d t\left(u_{1}, \ldots, u_{p}\right)=\int_{0}^{1} F_{t}^{*}\left(V_{t}\right\rfloor d \omega\right)\left(u_{1}, \ldots, u_{p}\right) d t \\
\quad=\int_{0}^{1} d \omega\left(V_{t} \circ F_{t}, F_{t_{*}} u_{1}, \ldots, F_{t_{*}} u_{p}\right) d t=\int_{0}^{1} d \omega\left(\frac{d F_{t}}{d t}, F_{t_{*}} u_{1}, \ldots, F_{t_{*}} u_{p}\right) d t=0
\end{aligned}
$$

since $F_{t}\left(\mathcal{F}_{q}\right)=\mathcal{F}_{q}$ and $d \omega \in \Omega^{p+1}(Q, \mathcal{F})$. This implies that $\left.\int_{0}^{1} F_{t}^{*}\left(V_{t}\right\rfloor d \omega\right) d t \in \Omega^{p}(Q, \mathcal{F})$.

Finally we obtain

$$
\omega-\left(F_{0}^{*} \circ \iota^{*}\right)(\omega)=\beta+d \alpha,
$$

where $\beta \in \Omega^{p}(Q, \mathcal{F})$ and $\left.\alpha=\int_{0}^{1} F_{t}^{*}\left(V_{t}\right\rfloor \omega\right) d t$. Thus

$$
[\omega]=\left[\left(F_{0}^{*} \iota^{*}(\omega)\right] \in H^{p}(\mathcal{F}) .\right.
$$

This implies that $F_{0}^{*} \circ \iota^{*}=i d_{H^{p}(\mathcal{F})}$.

On the other hand, $\iota^{*} \circ F_{0}^{*}=i d_{H^{p}(\mathcal{F} \cap S)}$, since $F_{0} \circ \iota=i d_{S}$. Thus $F_{0}^{*}$ is the required isomorphism between cohomology groups $H^{p}(\mathcal{F} \cap S)$ and $H^{p}(\mathcal{F})$.

3. Integrability of a characteristic distribution. Let $Q$ be a submanifold of a locally conformal symplectic manifold $(M, \Omega, \omega)$, $\operatorname{dim} M=2 n$. Let $\iota: Q \hookrightarrow M$ denote the standard inclusion of $Q$ in $M$. Let

$$
\left(T_{q} Q\right)^{\Omega}=\left\{v \in T_{q} M \mid \Omega(v, w)=0 \forall w \in T_{q} Q\right\} .
$$

We assume that $\operatorname{dim}\left(T_{q} Q\right)^{\Omega} \cap T_{q} Q$ is constant for every $q \in Q$. By $(T Q)^{\Omega} \cap T Q$ we denote the characteristic distribution $\bigcup_{q \in Q}\left(T_{q} Q\right)^{\Omega} \cap T_{q} Q$ which is a subbundle of the tangent bundle to $Q$. Now we prove

Proposition 3. The characteristic distribution $(T Q)^{\Omega} \cap T Q$ is involutive.

Proof. Let $X, Y$ be smooth sections of $(T Q)^{\Omega} \cap T Q$. We show that $[X, Y]$ is also a section of $(T Q)^{\Omega} \cap T Q$. By the well-known formula, for a smooth vector field $Z$ on $Q$ we have

$$
\begin{aligned}
d \Omega(X, Y, Z)= & X(\Omega(Y, Z))-Y(\Omega(X, Z))+Z(\Omega(X, Y)) \\
& +\Omega([X, Z], Y)-\Omega([X, Y], Z)-\Omega([Y, Z], X) \\
= & -\Omega([X, Y], Z),
\end{aligned}
$$

because $X, Y$ are smooth sections of $(T Q)^{\Omega} \cap T Q$. On the other hand $d \Omega=\omega \wedge \Omega$. Therefore

$$
d \Omega(X, Y, Z)=\omega(X) \Omega(Y, Z)+\omega(Y) \Omega(Z, X)+\omega(Z) \Omega(X, Y)=0 .
$$

Thus we obtain $\Omega([X, Y], Z)=0$ for every smooth vector field $Z$ on $Q$. On the other hand $[X, Y]$ is a section of $T Q$, since $X, Y$ are sections of $T Q$. Therefore $[X, Y]$ is a smooth section of $(T Q)^{\Omega} \cap T Q$.

4. Reduction of locally conformal symplectic forms. By Frobenius' theorem and Proposition 3, $(T Q)^{\Omega} \cap T Q$ is integrable and defines a foliation $\mathcal{F}$, which is called a characteristic foliation. Let $N=Q / \mathcal{F}$ be the quotient space obtained by identification of all points on a leaf. Assume that $N=Q / \mathcal{F}$ is a smooth manifold and the canonical 
projection $\pi: Q \rightarrow N=Q / \mathcal{F}$ is a submersion. If $\Omega$ is a symplectic form then there exists a symplectic structure $\tau$ on $N$ such that

$$
\pi^{*} \tau=\iota^{*} \Omega
$$

where $\iota: Q \hookrightarrow M$ denotes the standard inclusion of $Q$ in $M$ (see [13], [1], [2], [7], [12], [15], [4] and many others).

In [10] the reduction procedure for locally conformal symplectic manifolds that satisfies condition (3) is proposed.

A necessary and sufficient condition for existence of a conformal symplectic form on the reduced manifold $N=Q / \mathcal{F}$, which satisfies condition (3), is presented in the following theorem (see also Section 3 in [10]).

Proposition 4. Let $Q$ be a submanifold of a locally conformal symplectic structure $(M, \Omega, \omega)$, let $\iota: Q \hookrightarrow M$ denote the standard inclusion of $Q$ in $M$ and let $\mathcal{F}$ be the characteristic foliation of the characteristic distribution $T Q^{\Omega} \cap T Q$ of constant dimension. If $N=Q / \mathcal{F}$ is a manifold of dimension greater than 2 and the canonical projection $\pi: Q \rightarrow N=Q / \mathcal{F}$ is a submersion then there exists a locally conformal symplectic form $\tau$ on $N$ such that $\pi^{*} \tau=\iota^{*} \Omega$ if and only if

$$
\iota^{*} \omega(X)=0
$$

for every smooth section $X$ of $T Q^{\Omega} \cap T Q$.

Proof. Assume that there exists a locally conformal symplectic form $\tau$ on $N$ such that $\pi^{*} \tau=\iota^{*} \Omega$. Then

$$
\pi^{*} d \tau=\iota^{*} d \Omega=\iota^{*} \omega \wedge \iota^{*} \Omega .
$$

Therefore, for every smooth section $X$ of $T Q^{\Omega} \cap T Q$ we have

$$
\left.X\rfloor\left(\iota^{*} \omega \wedge \iota^{*} \Omega\right)=X\right\rfloor\left(\pi^{*} d \tau\right)=0,
$$

because $\pi_{*}(X)=0$. But $\iota^{*} \Omega \neq 0$ and $\left.X\right\rfloor \iota^{*} \Omega=0$, therefore $\iota^{*} \omega(X)=0$.

Now assume that $\iota^{*} \omega(X)=0$ for every smooth section $X$ of $T Q^{\Omega} \cap T Q$. Then

$$
\left.X\rfloor d \iota^{*} \Omega=X\right\rfloor\left(\iota^{*} \omega \wedge \iota^{*} \Omega\right)=0 .
$$

Hence

$$
\left.\left.L_{X} \iota^{*} \Omega=X\right\rfloor\left(d \iota^{*} \Omega\right)+d(X\rfloor \iota^{*} \Omega\right)=0
$$

for every smooth section $X$ of $T Q^{\Omega} \cap T Q$. Therefore $\Omega$ is constant on every leaf of the characteristic foliation $\mathcal{F}$. Now we define the form $\tau$ by the formula

$$
\pi^{*} \tau=\iota^{*} \Omega \text {. }
$$

$\tau$ is well-defined, because $\pi$ is a submersion. It is nondegenerate, because the kernel of $\iota^{*} \Omega$ is $T Q^{\Omega} \cap T Q=k e r \pi_{*}$. From the definition of $\tau$ we obtain

$$
\pi^{*} d \tau=d \iota^{*} \Omega=\iota^{*} \omega \wedge \iota^{*} \Omega=\iota^{*} \omega \wedge \pi^{*} \tau .
$$

We define $\alpha$ by the formula

$$
\pi^{*} \alpha=\iota^{*} \omega
$$

$\alpha$ is a well-defined closed 1 -form on $N$, because $\pi$ is a submersion and $\omega$ is closed. From (5) we have $d \tau=\alpha \wedge \tau$. 
Notice that a generic hypersurface on $M$ does not satisfy assumption (4) (even locally). EXAMPLE 1. Let $H$ be a smooth hypersurface on a locally conformal symplectic manifold $(M, \Omega, \omega)$.

By the Darboux theorem the germs at $q$ of $(M, \Omega, \omega)$ and $H$ are locally diffeomorphic to the germs at 0 of $\left(\mathbb{R}^{2 n}, f \sum_{i=1}^{n} d x_{i} \wedge d y_{i}, d f\right)$ and $\left\{(x, y) \in \mathbb{R}^{2 n}: x_{1}=0\right\}$, where $f$ is a smooth function-germ on $\mathbb{R}$ at 0 and $\operatorname{dim} M=2 n$.

Then the characteristic distribution $T\left\{(x, y) \in \mathbb{R}^{2 n}: x_{1}=0\right\}^{\Omega}$ is spanned by $\frac{\partial}{\partial y_{1}}$. The reduced manifold can be locally identified with $\left\{(x, y) \in \mathbb{R}^{2 n}: x_{1}=y_{1}=0\right\}$.

There exists a locally conformal symplectic structure $\tau$ on the reduced manifold satisfying condition (3) if and only if $\left.\frac{\partial f}{\partial y_{1}}\right|_{\left\{x_{1}=0\right\}}=0$.

In the next section we propose a procedure of reduction of locally conformal symplectic structures and find a sufficient and necessary condition for this reduction to hold in terms of a cohomology class of the restriction of $\omega$ to the coisotropic submanifold in the first cohomology group tangent to its characteristic foliation.

5. Reduction of locally conformal symplectic structures. Let $(M, \Omega, \omega)$ be a locally conformal symplectic manifold. Let $Q$ be submanifold of $M$, let $\iota: Q \hookrightarrow M$ denote the standard inclusion of $Q$ in $M$ and let $\mathcal{F}$ be the characteristic foliation of the characteristic distribution $T Q^{\Omega} \cap T Q$ of constant dimension smaller than $\operatorname{dim} Q$.

Proposition 5. If $\Omega^{\prime}$ is a locally conformal symplectic form conformally equivalent to $\Omega$ on $Q$ then the characteristic foliation $\mathcal{F}^{\prime}$ of $T Q^{\Omega^{\prime}} \cap T Q$ coincides with $\mathcal{F}$. If $\omega^{\prime}$ is the Lee form of $\Omega^{\prime}$ then $\left[\iota^{*} \omega\right]=\left[\iota^{*} \omega^{\prime}\right]$ in $H^{1}(\mathcal{F})$.

Proof. If $\Omega$ and $\Omega^{\prime}$ are conformally equivalent on $Q$ then there exists a positive function $f$ on $Q$ such that

$$
\iota^{*} \Omega=f \iota^{*} \Omega^{\prime}
$$

Thus it is obvious that $\mathcal{F}=\mathcal{F}^{\prime}$, since $T Q^{\Omega^{\prime}} \cap T Q=\operatorname{ker}^{*} \Omega^{\prime}=\operatorname{ker} \iota^{*} \Omega=T Q^{\Omega} \cap T Q$. Differentiating (6) we obtain

$$
\iota^{*} \omega \wedge \iota^{*} \Omega=f \iota^{*} \omega^{\prime} \wedge \iota^{*} \Omega^{\prime}+d f \wedge \iota^{*} \Omega^{\prime}
$$

Using (6) again we have

$$
\left(\iota^{*} \omega-\iota^{*} \omega^{\prime}-d(\ln f)\right) \wedge \iota^{*} \Omega^{\prime}=0
$$

Let $v$ be a vector tangent to a foliation. Then

$$
v\rfloor\left(\iota^{*} \omega-\iota^{*} \omega^{\prime}-d(\ln f)\right) \wedge \iota^{*} \Omega^{\prime}=0 .
$$

But $\iota^{*} \Omega^{\prime} \neq 0$ and $\left.v\right\rfloor \iota^{*} \Omega^{\prime}=0$, therefore

$$
\iota^{*} \omega(v)-\iota^{*} \omega^{\prime}(v)-d(\ln (f))(v)=0 .
$$

This implies that $\left[\iota^{*} \omega\right]=\left[\iota^{*} \omega^{\prime}\right]$ in $H^{1}(\mathcal{F})$.

Proposition 5 means that the cohomology class $\left[\iota^{*} \omega\right]$ in $H^{1}(\mathcal{F})$ is an invariant of the restriction of a locally conformal symplectic structure to $Q$. In the next theorem we use this class to state a necessary and sufficient condition for a reduced locally conformal symplectic structure to exist on a reduced manifold. 
THEOREM 2. Let $Q$ be a submanifold of a locally conformal symplectic manifold $(M, \Omega, \omega)$, let $\iota: Q \hookrightarrow M$ denote the standard inclusion of $Q$ in $M$ and let $\mathcal{F}$ be the characteristic foliation of the characteristic distribution $T Q^{\Omega} \cap T Q$ of constant dimension.

If $N=Q / \mathcal{F}$ is a manifold of dimension greater than 2 and the canonical projection $\pi: Q \rightarrow N=Q / \mathcal{F}$ is a submersion then there exist a locally conformal symplectic form $\tau$ on $N$ and a smooth positive function $f$ on $Q$ such that

$$
\pi^{*} \tau=f \iota^{*} \Omega
$$

if and only if $\left[\iota^{*} \omega\right]=0 \in H^{1}(\mathcal{F})$.

Proof. Assume that there exists a locally conformal symplectic form $\tau$ on $N$ and a positive smooth function $f$ on $Q$ such that $\pi^{*} \tau=f \iota^{*} \Omega$. Then

$$
\pi^{*} d \tau=d f \wedge \iota^{*} \Omega+f \iota^{*} d \Omega=f\left(d(\ln (f))+\iota^{*} \omega\right) \wedge \iota^{*} \Omega .
$$

Therefore, for any $q \in Q$ and any vector $v$ tangent to $\mathcal{F}_{q}$ we have

$$
\left.v\rfloor\left(\left(\iota^{*} \omega+d(\ln (f))\right) \wedge \iota^{*} \Omega\right)=v\right\rfloor\left(\frac{\pi^{*} d \tau}{f}\right)=0,
$$

since $\pi_{*}(v)=0$. But $\iota^{*} \Omega \neq 0$ and $\left.v\right\rfloor \iota^{*} \Omega=0$, therefore

$$
\iota^{*} \omega(v)+d(\ln (f))(v)=0
$$

for any $v$ tangent to $\mathcal{F}_{q}$. This implies that $\left[\iota^{*} \omega\right]=0 \in H^{1}(\mathcal{F})$.

Now assume that $\left[\iota^{*} \omega\right]=0 \in H^{1}(\mathcal{F})$. Then there exists a function $g$ on $Q$ such that $\iota^{*} \omega(v)=d g(v)$ for any $q \in Q$ and any vector $v$ tangent to $\mathcal{F}_{q}$. Thus

$$
\begin{gathered}
v\rfloor d\left(\exp (-g) \iota^{*} \Omega\right)= \\
\left.\exp (-g)\left(-d g(v)+\iota^{*} \omega(v)\right) \iota^{*} \Omega-\exp (-g)\left(-d g+\iota^{*} \omega\right) \wedge v\right\rfloor \iota^{*} \Omega=0 .
\end{gathered}
$$

Hence

$$
\left.\left.\mathcal{L}_{X} \exp (-g) \iota^{*} \Omega=X\right\rfloor d\left(\exp (-g) \iota^{*} \Omega\right)+d(X\rfloor \exp (-g) \iota^{*} \Omega\right)=0
$$

for every smooth section $X$ of $T Q^{\Omega} \cap T Q$. Therefore $\exp (-g) \iota^{*} \Omega$ is constant on every leaf of the characteristic foliation $\mathcal{F}$. Now we define the form $\tau$ by the formula

$$
\pi^{*} \tau=\exp (-g) \iota^{*} \Omega \text {. }
$$

$\tau$ is well-defined, because $\pi$ is a submersion. It is nondegenerate, because the kernel of $\exp (-g) \iota^{*} \Omega$ is $T Q^{\Omega} \cap T Q=\operatorname{ker} \pi_{*}$. From the definition of $\tau$ we obtain

$$
\pi^{*} d \tau=d\left(\exp (-g) \iota^{*} \Omega\right)=\left(\iota^{*} \omega-d g\right) \wedge \exp (-g) \iota^{*} \Omega=\left(\iota^{*} \omega-d g\right) \wedge \pi^{*} \tau .
$$

We define $\alpha$ by the formula

$$
\pi^{*} \alpha=\iota^{*} \omega-d g .
$$

$\alpha$ is a well-defined closed 1 -form on $N$, because $\pi$ is a submersion and $\omega$ is closed. From (8) we have $d \tau=\alpha \wedge \tau$.

By Proposition 5 and Theorem 2 it is easy to see that the reduction does not depend of the choice of a locally conformal symplectic form from the conformal equivalence class. Two locally conformal symplectic forms conformally equivalent on $Q$ are reduced to the same locally conformal symplectic structure on a reduced space. Thus Theorem 2 gives a procedure of reduction of locally conformal symplectic structures. 
Now we show how this procedure of reduction works. Any germ of a coisotropic submanifold can be reduced using the above procedure, since a locally conformal symplectic manifold is locally equivalent to a symplectic manifold. The obstruction to existence of a locally conformal structure on the reduced manifold is only global.

COROLlary 1. Let $Q$ be the germ at $q$ of a coisotropic submanifold of a locally conformal symplectic manifold $(M, \Omega, \omega)$, let $\iota: Q \hookrightarrow M$ denote the germ of the inclusion of $Q$ in $M$ and let $\mathcal{F}$ be the characteristic foliation of $T Q^{\Omega} \cap T Q=T Q^{\Omega}$.

Then there exists a germ of a locally conformal symplectic form $\tau$ on the germ of the reduced manifold $N=Q / \mathcal{F}$ and a germ of a smooth positive function $f$ on $Q$ such that $\pi^{*} \tau=f \iota^{*} \Omega$ where $\pi: Q \rightarrow N=Q / \mathcal{F}$ is the germ of the canonical projection.

\section{References}

[1] R. Abraham and J. E. Marsden, Foundations of Mechanics, 2nd ed., Benjamin/Cummings, Reading, 1978.

[2] V. I. Arnold, Mathematical Methods in Classical Mechanics, Springer-Verlag, 1978.

[3] A. Banyaga, Some properties of locally conformal symplectic structures, Comment. Math. Helv. 77 (2002), 383-398.

[4] W. Domitrz and S. Janeczko, Normal forms of symplectic structures on the stratified spaces, Colloquium Mathematicum 68 (1995), 101-119.

[5] W. Domitrz and S. Janeczko, On Martinet's singular symplectic structures, in: Singularities and Differential Equations, Banach Center Publications 33, 1996, 51-59.

[6] W. Domitrz, S. Janeczko and M. Zhitomirskii, Relative Poincaré lemma, contractibility, quasi-homogeneity and vector fields tangent to a singular variety, Illinois J. Math. 48 (2004), 803-835.

[7] V. Guillemin and S. Sternberg, Symplectic Techniques in Physics, 2nd ed., Cambridge University Press, Cambridge, 1990.

[8] S. Haller and T. Rybicki, On the group of diffeomorphisms preserving a locally conformal symplectic structure, Ann. Global. Anal. Geom. 17 (1999), 475-502.

[9] S. Haller and T. Rybicki, Integrability of Poisson algebra on a locally conformal symplectic structure, Rendiconti del Circolo Matematico di Palermo, Serie II, suppl. 63 (2000), 89-96.

[10] S. Haller and T. Rybicki, Reduction for locally conformal sympletic manifolds, J. Geom. Phys. 37 (2001), 262-271.

[11] H. C. Lee, A kind of even-dimensional differential geometry and its application to exterior calculus, Amer. J. Math. 65 (1943), 433-438.

[12] J. E. Marsden and T. Ratiu, Reduction of Poisson manifolds, Lett. Math. Phys. 11 (1986), 161-169.

[13] J. Marsden and A. Weinstein, Reduction of symplectic manifolds with symmetry, Rep. Mathematical Phys. 5 (1974), 121-130.

[14] D. McDuff and D. Salamon, Introduction to Symplectic Topology, Oxford University Press, 1995.

[15] J.-P. Ortega and T. Ratiu, Momentum Maps and Hamiltonian Reduction, Progress in Mathematics 222, Birkhäuser Boston, Boston, MA, 2004.

[16] I. Vaisman, Cohomology and Differential Forms, Pure and Applied Mathematics, Marcel Dekker, 1973. 
[17] I. Vaisman, Locally conformal symplectic manifolds, Internat. J. Math. \& Math. Sci. 8 (1985), 521-536.

[18] M. P. Wojtkowski and C. Liverani, Conformally symplectic dynamics and symmetry of the Lyapunov spectrum, Comm. Math. Phys. 194 (1998), 47-60. 
